\title{
Another Route to CP violation beyond the SM - the neutron EDM
}

\author{
Maurits van der Grinten* \\ Rutherford Appleton Laboratory/ University of Sussex \\ Chilton, Didcot OXON OX11 OQX,UK \\ E-mail: M.van-der-Grintenesussex.ac.uk
}

\begin{abstract}
The neutron electric dipole moment (EDM) experiment at the Institut Laue-Langevin (ILL) has completed data taking and subsequently the systematic errors have been evaluated. In this paper the experimental technique is discussed and results of the experiment are given. The experiment is based on a precision measurement of the Larmor precession frequency of neutrons in a magnetic field and employs a cohabiting mercury magnetometer. We have reached a final statistical precision of $1.5 \times 10^{-26} e \mathrm{~cm}$, the systematic potential for error, dominated by geometric phase shifts due to magnetic field inhomogeneities in the experiment, is found to be of the same order as the statistical precision obtained.
\end{abstract}

European Physical Society HEP2005 International Europhysics Conference on High Energy Physics EPS (July $21^{\text {st }}-27^{\text {th }} 2005$ ) in Lisboa, Portugal

\footnotetext{
* On behalf of the nEDM collaboration:

C.A. Baker, K.Green, M.G.D. van der Grinten, P.S. Iaydjiev, S.N. Ivanov and D.L. Wark Rutherford Appleton Laboratory, Chilton, Didcot, OX11 OQX UK

D.D.Doyle, P.G. Harris, D.J.R. May, J.M. Pendlebury, D.J. Richardson, D.B. Shiers and K.F. Smith University of Sussex, Falmer, Brighton BNI 9QH, UK

P. Geltenbort

Institut Laue-Langevin, BP156, F-38042 Grenoble, France
} 


\section{Introduction}

For particles to have electric dipole moments, the forces concerned in their structure must be asymmetric with regard to space-parity (P) and time reversal (T) [1]. Experimental observations of CP (and hence $\mathrm{T}$ ) violation, has thus far been found in the neutral kaon system [2] and in the decay of B mesons, and these seem to confirm our picture of CP violation in the Standard Model of particle physics. CP violation is believed to be responsible for the baryon asymmetry of the Universe but the CP violation found in the Standard Model is many orders of magnitude too small to account for the observed baryon asymmetry. This leaves open a wide range of possibilities for competing theories attempting to explain the origin of $\mathrm{CP}$ violation. Extensions to the Standard Model of the electroweak interaction, such as additional Higgs fields, right-handed currents or supersymmetric partners typically give rise to dipole contributions which are of order $10^{-26}$ to $10^{-28} e \mathrm{~cm}$. Dipole moments of this size might also come from $\mathrm{CP}$ violation in the sector of the strong interaction. Experimental measurements of particle EDMs, and in particular that of the neutron, are providing some of the strongest additional constraints on these theories. The current experimental limit on the neutron EDM is set by our collaboration to $\left|d_{n}\right|<6.3 \times e \mathrm{~cm}(90 \% \mathrm{CL})$ [3].

\section{Experimental techniques}

The Ramsey resonance technique is used to measure, with very high precision, the Larmor precession frequency of spin polarised ultracold neutrons (UCN) in a weak magnetic field. The precession frequency will change in the presence of an electric field if the neutron has an EDM. The EDM experiment incorporates a magnetometer based on atomic ${ }^{199} \mathrm{Hg}$ stored simultaneously in the same cell as the neutrons [4]. This system provides us with a magnetometer probing the UCN storage cell with an accuracy of $1 \mathrm{nG}$. The measurement is made with UCN stored in a cell permeated by $\mathbf{E}$ - and $\mathbf{B}$-fields. The terms $-\boldsymbol{\mu}_{\mathbf{n}} \cdot \mathbf{B}$ and $-\mathbf{d}_{\mathbf{n}} \cdot \mathbf{E}$ are added to the Hamiltonian determining the states of the neutron. The difference in Larmor frequencies between parallel and antiparallel $\mathbf{E}$ and $\mathbf{B}$ fields relate to $d_{\mathrm{n}}$ via

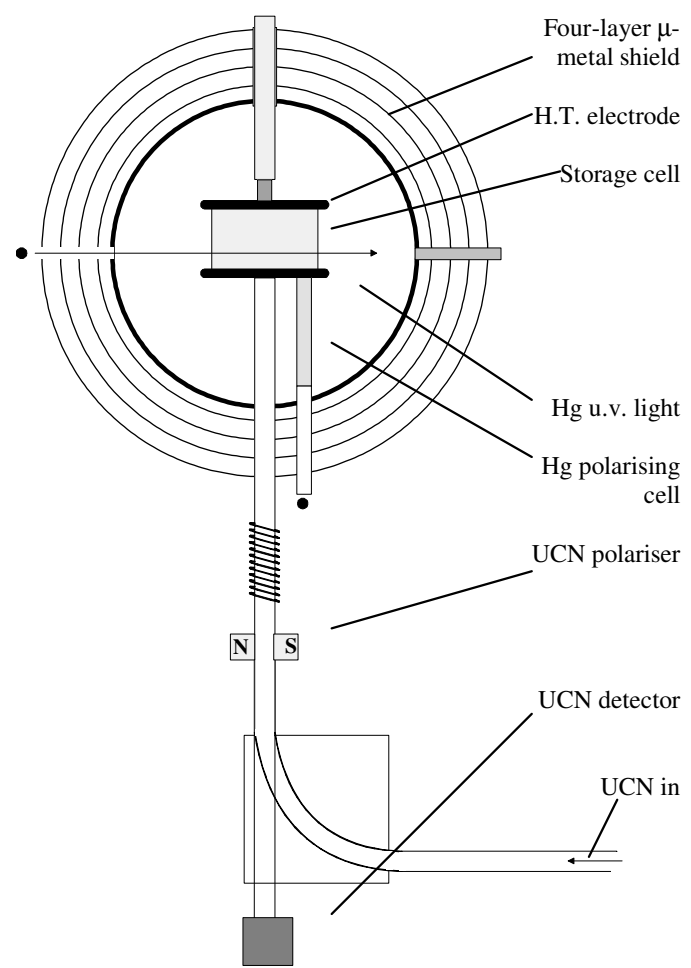

Fig. 1. The experimental set-up at ILL-Grenoble to measure the neutron EDM 
$h\left(v_{\uparrow \uparrow}-v_{\uparrow} \downarrow\right)=4 \mathbf{d}_{\mathrm{n}} . \mathbf{E}$. Thus, the goal is to measure any shift in the transition frequency $v$ when a strong $\mathbf{E}$ field is reversed relative to $\mathbf{B}$.

Fig. 1 shows the set-up of the experiment. Neutrons are fed from the UCN source PF2 at the ILL-Grenoble into the 20 litre storage cell placed in a $10 \mathrm{mG}$ magnetic field where the actual Ramsey measurement takes place. The polarisation $P$ of the neutrons is given by the quantity $\alpha=P^{2}$. The statistical precision of a measurement is

$$
\sigma\left(d_{n}\right)=\frac{\hbar}{2 \alpha E T \sqrt{N}} .
$$

Near the end of the experiment we reached values of $\alpha=0.7$, the electric field strength $E=$ $11 \mathrm{kV} / \mathrm{cm}$, the storage time $T=130$, and $N=18000$ neutrons counted per batch. From one day of data $\sigma\left(d_{\mathrm{n}}\right)$ was then about $1.5 \times 10^{-25} e \mathrm{~cm}$.

\section{Acquired data}

The experiment has been acquiring data for a total of six years. Fig. 2 shows the statistical precision that we have accumulated over this period. The precision with which the EDM measurements have been made has improved steadily over the years. As the experiment was carried out techniques have been developed and incorporated in the experiment that allowed e.g. to increase the electric field strength and

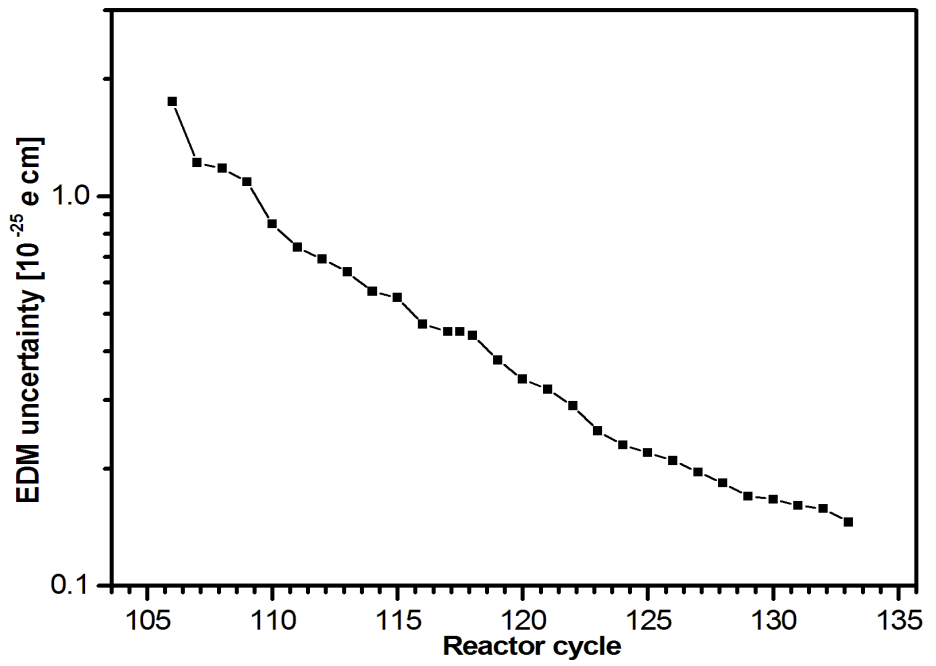

Fig. 2. The statistical precision on the neutron EDM accumulated per reactor cycle. The period covers the full six years of the experiments running time. the storage potential of the cell. As shown in Fig. 2, the resulting final statistical sensitivity that we obtained in our measurement is $1.5 \times 10^{-26} e \mathrm{~cm}$.

\section{Systematic effects in the experiment}

There are a number of effects that can alter the precession frequency of the neutron and generate a false EDM signal or shift any measured EDM signal. E.g. effects such as leakage currents passing through the cell could off-set the magnetic field in a direction related to the 
direction of the electric field and hence be interpreted as a true EDM signal. Most of these effects are suppressed by the configuration of the experiment to a level well below $10^{-26} e \mathrm{~cm}$, i.e. a level well below the obtained statistical precision. However there is one mechanism, the geometric phase effect described in [5], that can generate EDM signals big enough to affect our result. This mechanism stems from a combination of the so-called $\mathbf{v} \times \mathbf{E}$ effect and a vertical magnetic field gradient present in the storage cell which results in a magnetic field, as perceived by the neutrons, that is rotating in the horizontal plane. The rotating magnetic field will, through the Ramsey-Bloch-Siegert mechanism, shift the precession frequency of the neutron in response to an applied electric field and thus generate a false EDM signal. We have indeed found this effect by analysing the data as function of the vertical magnetic field gradients present in the experiment. The effect introduces a possible systematic error that is of the same order as the statistical uncertainty and presents our leading systematic error.

\section{Conclusions}

We have made a measurement of the $\mathrm{nEDM}$ by making a precision measurement of the Larmor precession of a neutron stored in a magnetic field aligned to an external electric field. The data taking is completed and studies have been carried out to identify and quantify the systematic effects present in the experiment. The final statistical uncertainty that is obtained is $1.5 \times 10^{-26} e \mathrm{~cm}$. The leading systematic effect is well understood and gives rise to a possible systematic error that is of the same order in size as the statistical uncertainty.

\section{Acknowledgments}

This work has been funded by a grant from the U.K. Particle Physics and Astronomy Research Council (PPARC). S.N.I. and P.S.I. have carried out this work on leave of absence from PNPI, St Petersburg, Russia and INRE, Sofia, Bulgaria respectively.

\section{References}

[1] S.M. Barr Int. J. Mod. Phys. A 8, 209 (1993)

[2] J.H. Christenson, J.W. Cronin, V.L. Fitch and R. Turlay. Phys. Rev. Lett., 13 (1964) 138-140

[3] P.G. Harris, C.A. Baker, K. Green, P. Iaydjiev, S.N. Ivanov, D.J.R May, J.M. Pendlebury, D. Shiers, K.F. Smith, M. van der Grinten and P. Geltenbort, Phys. Rev. Lett. 82-5 (1999) 904 907.

[4] K. Green, P.G. Harris, P. Iaydjiev, D.J.R. May, J.M. Pendlebury, K.F. Smith, M. van der Grinten, P. Geltenbort and S. Ivanov, Nucl. Instr. Meth.-B 404 2-3 (1998) 381-393.

[5] J.M. Pendlebury, W. Heil, Yu. Sobolev, P.G. Harris, J.D. Richardson, R.J. Baskin, D.D. Doyle, P. Geltenbort, K. Green, M.G.D. van der Grinten, P.S. Iaydjiev, S.N. Ivanov, D.J.R. May, and K.F. Smith, Phys. Rev. A 70 (2004) 032102 